\title{
La campaña de las autonómicas catalanas de 2006 en la prensa de referencia
}

\section{Elena Cebrián Guinovart}

Profesora de Periodismo Especializado

Universitat CEU Abat Oliba

\section{Resumen:}

El hecho de que la sociedad catalana estuviera profundamente desencantada con la política y que los partidos aspiraran a fortalecer sus resultados en las urnas para evitar la inestabilidad de la legislatura anterior, dota a la campaña de las Elecciones Autonómicas catalanas, del 1 de noviembre de 2006, de especial influencia en el voto. Este trabajo examina, mediante una análisis de contenido cuantitativo, la presentación de esa campaña en los cuatro periódicos más influyentes y demuestra que la mayor parte de la cobertura total se centró en las actividades de los partidos, que la agenda que siguieron los diarios no coincidió con las preocupaciones declaradas, ni con el voto emitido por los ciudadanos, y que se presentó una campaña fuertemente espectacularizada, muy personalizada y con más ataques que propuestas políticas.

Palabras clave:

Campaña electoral - Partidos políticos - Agenda informativa - Personalización política - Política espectáculo - Argumentación política

\section{Abstract:}

The fact that Catalan society was deeply disenchanted with politics and that the parties aspired to get better results at the polls, gives the Electoral campaign for Catalan Parliament Elections on $1^{\text {st }}$ November, 2006 a special influence in vote decision. Through a quantitative content analysis, this paper studies the presentation of that campaign in the four most influential newspapers, and proves that most of the total coverage was centred in the activities of the different parties, that social interests and media agenda 
differed greatly, and that the press showed a very personal and spectacular campaign with far more attacks than political proposals.

\section{Keywords:}

Electoral campaign - Political parties - Media Agenda - Personalised politics - Spectacularised Politics Political line of argument

\section{Introducción}

Antes del comienzo de la campaña de la Elecciones Autonómicas de Cataluña del 1 de noviembre de 2006, el desencanto de los electores con la política era manifiesto: en octubre más de la mitad de los catalanes -el 56,8\%- se declaraban "Poco" o "Nada" interesados en política (Generalitat de Cataluña, 2006a: 11), más de dos terceras partes -68,6\%- decían estar "Mas bien de acuerdo" con la afirmación "Los políticos sólo buscan su propio beneficio" (Generalitat de Cataluña, 2006a: 14), y tres cuartas partes -el 76,1\%-declaraban no creer que los políticos tuvieran en cuenta lo que piensa la gente (Generalitat de Cataluña, 2006a: 13). En contraste con este desencanto los principales partidos necesitaban dinamizar al electorado para, bien alcanzar la mayoría suficiente que les permitiera gobernar, bien mejorar sus resultados fortaleciendo su posición de cara al reparto de poder en los posibles pactos postelectorales. La conjunción de las dos situaciones indica que la campaña oficial se convirtió, en el caso de las elecciones catalanas de 2006, en un "momento simbólico privilegiado" en que los partidos actuaron para modificar las actitudes y los comportamientos políticos en un lapso breve (Gerstlé, 2005:123). Una afirmación que refuerza el hecho de que el $24 \%$ de los electores y el $33 \%$ de los abstencionistas dijeran haber decidido durante la campaña lo que harían el 1 de noviembre (CIDE-INCAS, 2007:9).

Este trabajo examina la representación que de esa campaña hizo la prensa de referencia, considerando que la información periodística fue una de las influencias principales: el $72,1 \%$ de los catalanes siguió la campaña a través de televisión o radio y el $57,2 \%$ leyó artículos o reportajes en prensa sobre las elecciones, frente al 36,3\% que leyó programas electorales o el 7,5\% que asistió a mítines u otras reuniones políticas (Generalitat de Cataluña, 2006b: 39). Si bien los ciudadanos siguieron más la campaña a través de la televisión o la radio que de los periódicos, se ha preferido analizar la representación de la campaña en la prensa considerando que, frente al impacto y el predominio de lo audiovisual, el periódico mantiene su estatus de medio de referencia al permitir un tratamiento cualitativamente mejor ${ }^{1}$.

El análisis se ha efectuado en los diarios de mayor penetración e influencia en el ámbito catalán - La Vanguardia y El Periódico de Catalunya- y las ediciones catalanas de los dos de mayor penetración

\footnotetext{
${ }^{1}$ Según Alejandro Muñoz Alonso los medios escritos "son los únicos capaces de dar cuenta en profundidad y desde todos los ángulos de los acontecimientos que se producen, los únicos en condiciones de aportar el contexto y el análisis sin los cuales las informaciones resultan imposibles de comprender" (MuÑOZ Alonso, 1997: 4).
} 
en el nacional -El País y $E l M u n d o^{2}$, y ha consistido en un análisis cuantitativo de toda la información electoral contenida en los 15 números que cada una de las cuatro cabeceras publicó durante la campaña oficial- entre el 16 y el 31 de octubre de 2006.

Los datos se recogieron en una base de datos elaborada con el programa Microsoft Excel diseñada con la estructura que se muestra a continuación:

Figura 1

Modelo de la tabla para la recogida de datos

\begin{tabular}{|l|l|l|l|}
\hline \multirow{2}{*}{ DATOS GENERALES } & Identificador & \multicolumn{2}{|l|}{} \\
\cline { 2 - 4 } & Diario & El País & La Vanguardia \\
\cline { 2 - 4 } & & El Mundo & El Periódico de Catalunya \\
\cline { 2 - 4 } & Fecha & & \\
\cline { 2 - 4 } & Página & & \\
\cline { 2 - 4 } & Titular & & \\
\cline { 2 - 4 } & Tamaño & & \\
\hline
\end{tabular}

\begin{tabular}{|l|l|l|l|}
\hline CONTENIDO & Asunto & Contexto & Campaña \\
\cline { 3 - 4 } & \multirow{3}{*}{ Partido } & CiU & PSC \\
\cline { 3 - 4 } & ERC & ICV-EUiA \\
\cline { 3 - 4 } & PP & C-CdC \\
\cline { 2 - 4 } & Otros & Genérico \\
\cline { 2 - 4 } Protagonista & Candidato & Partido catalán \\
\cline { 3 - 4 } & & Líder nacional \\
\cline { 3 - 4 } & & Partido nacional \\
\hline
\end{tabular}

${ }^{2}$ El resumen general del EGM de febrero a noviembre de 2006 -que incluye los días de la campaña- determina que el diario general nacional más leído es El País, con 2.058.000 lectores diarios, y El Mundo el segundo con 1.338.000. Entre las cabeceras regionales catalanas el EGM muestra que el más leído es El Periódico con 789.000 lectores diarios, seguido de La Vanguardia con 697.000 (Estudio General de Medios, 2006). Unos datos que completa el Barómetro de Opinión política de la Generalitat de Cataluña de Octubre de 2006, en el que según las respuesta a la pregunta 16c. "I quin diari acostuma a llegir amb més freqüència?" el primer y segundo diario más leídos en Cataluña son La Vanguardia (27,6\% de los encuestados) y la edición en catalán de $E l$ Periódico de Catalunya (18,5 \%), y de las cabeceras nacionales El País (9,5\%) y El Mundo (1,7\%). (Generalitat de Catalunya, 2006a:13). Además el estudio sociológico sobre el voto en las elecciones catalanas elaborado por CIDE-INCAS muestra que $L a$ Vanguardia y El Periódico son los diarios a través de los que se informaron preferentemente un mayor número de electores (CIDEINCAS, 2006:5). 


\begin{tabular}{|c|c|c|}
\hline \multirow[t]{4}{*}{ Acción } & \multirow[t]{4}{*}{ Descripción } & Afirmación \\
\hline & & Ataque \\
\hline & & Defensa \\
\hline & & Propuesta \\
\hline Enfoque & Política real & Política espectáculo \\
\hline \multirow[t]{13}{*}{ Tema } & Cultura & Acuerdos postelectorales \\
\hline & Economía & Campaña \\
\hline & Educación & Candidato \\
\hline & Infraestructuras & Partido \\
\hline & Inmigración & \\
\hline & Medioambiente & \\
\hline & Nacionalismo & \\
\hline & NNTT & \\
\hline & Salud & \\
\hline & Seguridad & \\
\hline & Social & \\
\hline & Vivienda & \\
\hline & Tripartito & \\
\hline
\end{tabular}

En el apartado "Datos generales" se consignó la información relacionada con las características formales o la ubicación de cada texto.

Los campos "Identificador" y “Tamaño" han proporcionado las unidades de medida del análisis. En "Identificador" se asignaba un número consecutivo a cada texto, y en "Tamaño" se registraba la superficie de cada uno en módulos reticulares ${ }^{3}$. Se introdujeron los dos criterios en la base de datos, puesto

${ }^{3}$ Jesús Zorrilla explica que, desde el punto de vista de la arquitectura de la página, "los módulos son los bloques rectangulares o cuadrangulares en los que esta se divide para su posterior relleno con material informativo” (Zorrilla, 1999:107), y define la retícula de una publicación como "la estructura casi invisible que subyace en las páginas de cualquier publicación bien diseñada" (Zorrilla, 1999:47). Se ha elegido esta unidad frente a otras posibles para la medición cuantitativa porque -pese a las diferencias entre las estructuras modulares de cada uno de los diarios analizados- organiza y determina el espacio de la información en las páginas del periódico atendiendo a criterios de relevancia informativa: según Zorrilla " Los módulos ayudan a racionalizar el espacio de una página . La gradación de la importancia de las informaciones implícita en la extensión del módulo, ayuda al lector a diferenciar las informaciones principales de las secundarias" (Zorrilla, 1999:52). 
que para algunos de los aspectos analizados el resultado era más significativo desde el punto de vista del volumen total del tratamiento -los módulos reticulares- y en otros lo significativo es el número de mensajes -el número de textos.

“Fecha”, "Página” y “Titular” se registraron como criterios para la localización de los textos junto con "Diario", si bien este último también se ha utilizado como una de las variables de referencia del trabajo: El País y El Mundo constituyen la categoría "Prensa nacional" y La Vanguardia y El Periódico la categoría "Prensa Catalana". Comparando el tipo de campaña presentado en una y otra categoría se buscaban diferencias en el enfoque determinadas por el ámbito geográfico.

En el apartado "Contenido" se recogieron los datos sobre los aspectos objeto del análisis: "Asunto" diferenciaba si el texto informaba sobre actos de la campaña de los partidos o sobre algún aspecto que permitiera contextualizar las propuestas de los partidos, la situación política de Cataluña, o la propia campaña. Como "Campaña" se han considerado las actividades electoralistas de los partidos con perspectiva amplia: tanto en los que las formaciones políticas llevan la iniciativa como en los que la iniciativa era de los medios. Considerar parte de la campaña las iniciativas de los medios que implican una participación activa de los candidatos o los partidos, se ajusta a la realidad vigente en la que, según explica Wolton, los políticos conscientes del poder de los medios responden a las iniciativas de estos como peaje de la visibilidad que les proporcionarán ${ }^{4}$.

Actos de campaña en que la iniciativa la llevaban los partidos son los mítines, los encuentros con los militantes, las reuniones con colectivos sociales a los que pedir el voto, o diferentes visitas con fines electoralistas. En cambio son actos de campaña incitados por los medios la participación de los candidatos en debates electorales o en otro tipo de enfrentamientos dialécticos, la concesión de entrevistas por parte de sus esposas o parejas, la respuesta de ellos mismos a cuestionarios de carácter político o $\mathrm{no}^{5}$, o la participación en entrevistas- tertulia con algún personaje relevante de la sociedad o la cultura catalana $^{6}$.

4 "El político sufre la presión del acontecimiento y la del cortejo de periodistas. Estos comentan a diario, encuentran significados ocultos a las estrategias improbables, y dudan de la capacidad de acción de los políticos, que se ven entonces obligados a correr de emisoras de radio a platós de televisión para responder al los rumores, confirmar algunos, desmentir otros, desmarcarse de quienes les hacen la competencia, cuidar su propia imagen, comprometer el futuro y no dar la impresión de ser incapaces de enfrentarse al presente" (Wolton, 1999: 150).

${ }^{5}$ Con carácter político, El Periódico de Catalunya publicó regularmente durante la campaña la polémica periodística "La pregunta del baròmetre" en la que presentaba la respuesta de los cabeza de lista de PSC, CiU, ERC, PP e ICV-EUiA a una pregunta sobre la que también se había encuestado a la sociedad catalana. Junto al infográfico que mostraba los porcentajes de respuesta de los encuestados se publicaba un párrafo en el que cada candidato explicaba la posición de su partido ante el asunto. Menos político es el carácter de dos series publicada por La Vanguardia: en la primera los cinco cabezas de lista mencionados, respondían 
El resto del análisis que a continuación se expone sólo se desarrolló en el caso de los textos que se clasificaron como "Campaña". "Partido" atribuía la información a su principal protagonista. Se consideraron los seis partidos que el 1 de noviembre obtuvieron representación en el Parlamento de Cataluña: Convergencia i Unió (CiU), Partit dels Socialistes de Catalunya (PSC), Esquerra Republicana de Catalunya (ERC), Partido Popular (PP), Iniciativa per Catalunya-Verds Ezquerra Unida i Alternativa (ICV-EUiA) y Ciutadans Partido de la Ciudadanía (C-PdC). Además se añadió una entrada para los textos que trataban partidos que no obtuvieron representación y otra para los que se ocupaban de los partidos de modo genérico o presentaban a varias formaciones de manera indiferenciada. Esta variable mostraría en que proporción se informaba de cada partido, a la vez que cruzada con otras detectaría diferencias en la presentación que los diarios hicieron de la campañas de las distintas formaciones.

En el campo "Protagonista" se han clasificado los textos dependiendo de si se centraban en el cabeza de lista -“Candidato"- o en el resto del partido considerado individual o colectivamente. En el caso de los textos que consideran partidos con una extensión nacional -PSC, PP, o ICV-EUiA se ha distinguido si la información se encarnaba en el partido en Cataluña, el partido en el resto de España, o el líder nacional del partido. Lo que se buscaba era conocer el grado de personalización entendida como la atención preferente en las personalidades frente a las ideas ${ }^{7}$, así como el grado de implicación en la campaña catalana de partidos y líderes nacionales en los casos en que los hubiera.

El campo "Acción” ha servido para analizar argumentalmente los mensajes de los partidos en la campaña. Para ello se valoró, en primer lugar, si el mensaje contenido en el titular era una toma de palabra del propio partido ${ }^{8}$, o si era el diario el que explicaba el mensaje con carácter informativo ${ }^{9}$. Los casos en que los diarios tomaron la palabra se anotaron como "Descripción”, mientras que en la toma

a un cuestionario del cocinero Santi Santamaría, y en la segunda mostraban y comentaban su reloj, sus zapatos, y dibujaban una escena marítima con el fin de que el diario analizara su personalidad.

${ }^{6}$ La Vanguardia publicó en los quince días de campaña series en las que los cabezas de lista de CiU, PSC, ERC, PP e ICVEUiA eran entrevistados por el economista Xavier Sala i Martín, o conversaban informalmente tomando el aperitivo con el periodista Joan de Sagarra.

Swanson explica que los medios "tienden a presentar las noticias políticas centrando la atención en las personalidades y no en las ideas. Los partidos políticos pueden verse inducidos a responder sacando al frente a los líderes o candidatos que queden especialmente atractivos e irresistibles en las imágenes de televisión y en otros medios" (Swanson, 1995: 19-20). En el mismo sentido Berrocal matiza que este fenómeno ha modificado la retórica del discurso político "Los líderes, a falta de proyectos políticos diferenciados, emiten imágenes que los medios recogen y presentan a la opinión pública” convirtiéndose en actores de la política. (Berrocal, 2004: 61)

${ }^{8}$ Por ejemplo son titulares en los que los partidos toman la palabra "Mas insta a ERC a usar 'la llave' esta vez para hacer presidente a 'un nacionalista”' (El Mundo, 23 de octubre, página 23) o "Montilla acusa a Más de haber dejado un 'agujero negro' del 1.000 millones de euros en la Generalitat" (El País, 17 de octubre, página 28)

${ }^{9}$ Son titulares en los que el diario explica un hecho "Piqué entrega carnets a 200 nuevos afiliados del PP catalán" (El País, 19 de octubre, página 36), o "CiU contacta con Puicercós al margen de Carod tras observar un repunte de ERC en los sondeos" (El Mundo, 28 de octubre, página 27). 
de palabra de los partidos se distinguió si el argumento era una afirmación, un ataque, una defensa o una propuesta. Siguiendo el análisis dialéctico al que Benoit, Blaney y Pier sometieron a la campaña presidencial americana de 1996 se ha considerado un ataque "criticar a los candidatos rivales por sus cualidades negativas o por acciones discutibles"10 y una defensa "responder a las críticas de otros" 11 (Benoit, Blaney, Pier, 1998: 22). "Propuesta"12 y "Afirmación"13 se han utilizado para consignar las promesas electorales que estos autores consideran una declaración política del tipo "Planes de futuro" y "Objetivos generales" (Benoit, Blaney, Pier, 1998: 25).

El análisis argumental efectuado en este punto busca saber si los diarios examinados seleccionaron asuntos que se ajustaran a lógicas de conflicto o dramatismo buscando información más atractiva para la audiencia, o asuntos afines a las dinámicas propias de la política democrática como la negociación o el compromiso (Swanson 1995: 13-14 y Muñoz-Alonso 1999: 42). Los mensajes del tipo "Propuesta" o “Afirmación" corresponden a la lógica política, y los del tipo "Ataque” o "Defensa” a la lógica mediática.

"Enfoque" ha servido para distinguir el punto de vista desde el que la información miraba el acontecimiento político. Se clasificaron como "Política real" los textos que proporcionaban una descripción de hechos políticos junto con los elementos de juicio necesarios para contextualizarlos, mientras que como "Política espectáculo" se clasificaron los que ofrecían visiones cínicas y negativas, enfoques personalistas, informaciones parciales o narraciones teatralizadas de la campaña (Berrocal, Abad, Cebrián y Pedreira, 2003: 281) $)^{14}$.

Por último en el campo "Tema" se clasificaron los textos atendiendo al principal contenido que trataron. Este fue un registro de carácter abierto en el que, al terminar el análisis todos los temas encontrados se agruparon en 17, que a su vez se clasificaron en dos grandes categorías: "Acción política" y "Representación política". La categoría de "Acción política" contiene 13 temas que se ajustan a los campos de la gestión política tradicional: "Cultura”, "Economía”, "Educación”, "Infraestructuras”, "In-

${ }^{10}$ Contiene un Ataque, por ejemplo, el titular "Carod-Rovira censura la poca ética de la campaña de CiU y de su DVD 'infame”" (El Mundo 16 de octubre, página 22)

11 "Llamazares replica a Montilla que el Govern Tripartito 'no es historia"” contiene una defensa (El Mundo, 28 de octubre, página 26)

${ }^{12}$ El titular "Piqué promete acabar con el déficit de mossos y equiparar la cifra de agentes a la media europea" (La Vanguardia, 25 de octubre, página 16) contiene una Propuesta

${ }^{13}$ Hay un ejemplo de Afirmación en el titular " Rajoy afirma que només el PP pot garantir 'un canvi polític”" (El Periódico de Catalunya, 31 de octubre, página 9 suplemento Autonòmiques 2006)

${ }^{14}$ Son ejemplos de temas de "Política espectáculo" los textos titulados "Un primo hermano de Maragall hace público su apoyo a la candidatura de Artur Mas" (El Mundo, 19 octubre, página 27), "Los candidatos discrepan al vaticinar el resultado del MadridBarça” (El País, 22 de octubre, página 34), "La publicidad de Mas interrumpe a Montilla” (La Vanguardia, 17 de octubre, página 17), o "Una caniche enana, vestida con camiseta y tejanos, aspira a convertirse en mascota del PP catalán" (El Mundo, 30 de octubre, 17 octubre, página 22). 
migración", "Medioambiente”, “Nacionalismo”, "Nuevas tecnologías”, "Salud", "Seguridad”, "Social”, "Vivienda" y "Tripartito". La categoría "Representación política” contiene cuatro temas -“Acuerdos postelectorales", "Campaña", "Candidato" y "Partido"- que se ocupan de asuntos relacionados con la visualización de la política o sus personajes ${ }^{15}$.

Los datos obtenidos en este análisis han permitido conocer tres aspectos sobre la presentación de la campaña de los partidos hecha por la prensa: el grado en que los diarios informaron sobre actos de los partidos o proporcionaron contexto a sus lectores, la agenda que los periódicos siguieron para presentarla, y el tipo de campaña mostraron. De cada uno de los tres aspectos se exponen a continuación los resultados totales y los que permiten observar las diferencias en el tratamiento del tema según ámbito geográfico o entre partidos.

\section{Contenido de la información electoral}

El reflejo de la campaña de los partidos - como muestra la tabla I- ocupa más de la mitad de toda la información electoral publicada a lo largo de la quincena de la campaña oficial, mientras que la explicación o contextualización de la propia campaña supone un $42 \%$.

Tabla I

Superficie de información dedicada a cada asunto

\begin{tabular}{|l|c|c|}
\cline { 2 - 3 } \multicolumn{1}{c|}{} & Módulos & (\%) \\
\hline Campaña & 19.032 & 58 \\
\hline Contexto & 14.004 & 42 \\
\hline Total & 33.036 & - \\
\hline
\end{tabular}

Las cifras totales de la tabla II muestran como globalmente los dos periódicos catalanes considerados informaron con mayor extensión sobre la campaña electoral: los 23.996 módulos que le dedicaron suponen el $73 \%$ frente al $27 \%$ que suponen los 9.040 módulos que le dedicaron los dos diarios nacionales.

${ }^{15}$ El texto "De Mel Gibson a Josep Lluís Carod-Rovira” (La Vanguardia, 17 octubre, página 17), que informa de que responsable de la puesta en escena de la campaña de ERC es el escenográfo de la película Braveheart, es un ejemplo del tema "Campaña". Las diversas entrevistas con las esposas o parejas de los candidatos, o las respuestas de los propios candidatos a cuestionarios de carácter absolutamente personal que los cuatro diarios publicaron en distintos momentos de la quincena de la campaña son ejemplos del tema "Candidato". Y el texto "Ciutadans irá a las municipales" (La Vanguardia, 18 de octubre, página19) es un ejemplo del tema "Partido" al centrarse en un asunto relacionado con el futuro de esta formación. 
Tabla II

Diferencias en la superficie dedicada a cada asunto según el lugar de edición

\begin{tabular}{|l|c|c|c|c|}
\cline { 2 - 5 } \multicolumn{1}{c|}{} & \multicolumn{2}{c|}{ Prensa Nacional } & \multicolumn{2}{c|}{ Prensa Catalana } \\
\cline { 2 - 5 } \multicolumn{1}{c|}{} & Módulos & $\mathbf{( \% )}$ & Módulos & (\%) \\
\hline Campaña & 5.466 & 60 & 13.566 & 57 \\
\hline Contexto & 3.574 & 40 & 10.430 & 43 \\
\hline Total & 9.040 & - & 23.996 & - \\
\hline
\end{tabular}

Tanto los nacionales como los catalanes -según la tabla II- dedicaron más espacio a las actividades de los partidos que a contextualizar la campaña, si bien la prensa catalana contextualiza ligeramente más que la nacional.

\section{Agenda al informar sobre la campaña de los partidos: temario y atención por partidos}

\subsection{Temario}

El asunto que más veces trataron los diarios analizados al informar de las actividades electoralistas de los partidos fue la propia campaña: algo más de una tercera parte de los textos, según muestra la tabla III. Los siguientes temas más frecuentes son los posibles pactos una vez celebradas las elecciones $-15 \%-$ diversas concreciones del nacionalismo catalán $-12 \%-$ y algún aspecto de los cabezas de lista de los partidos $-11 \%$. Ninguno de los demás temas asuntos tratados tiene una presencia relevante en la agenda temática, puesto que ninguno es supera el $5 \%$ de presencia.

Tabla III

Temario de las informaciones sobre actividades de los partidos

\begin{tabular}{|l|c|c|}
\cline { 2 - 3 } \multicolumn{1}{c|}{} & Textos & (\%) \\
\hline Cultura & 5 & 0 \\
\hline Economía & 35 & 4 \\
\hline Educación & 6 & 1 \\
\hline Infraestructuras & 19 & 2 \\
\hline Inmigración & 29 & 3 \\
\hline Medioambiente & 5 & 0 \\
\hline Nacionalismo & 122 & 12 \\
\hline NNTT & 2 & 0 \\
\hline
\end{tabular}




\begin{tabular}{|l|c|c|}
\cline { 2 - 3 } \multicolumn{1}{c|}{} & Textos & (\%) \\
\hline Salud & 4 & 0 \\
\hline Seguridad & 15 & 2 \\
\hline Social & 33 & 3 \\
\hline Vivienda & 2 & 0 \\
\hline Tripartito & 21 & 2 \\
\hline \multicolumn{2}{|c|}{} & $(29)$ \\
\hline Acuerdos postelectorales & $\mathbf{1 5 0}$ & $\mathbf{1 5}$ \\
\hline Campaña & $\mathbf{3 8 8}$ & $\mathbf{3 9}$ \\
\hline Candidato & $\mathbf{1 0 6}$ & $\mathbf{1 1}$ \\
\hline Partido & $\mathbf{4 6}$ & $\mathbf{5}$ \\
\hline \multicolumn{2}{|c|}{} & \\
\hline Total & 988 & - \\
\hline
\end{tabular}

La segunda conclusión relevante de la tabla III aparece al agrupar los temas en las categorías de Acción política (en blanco en la tabla) y Representación política (en gris en la tabla). El grueso de la información sobre la campaña se ocupó de aspectos de Representación política -más dos tercios de todos los textos- mientras que los temas de Acción política no llegan al 30\%.

Comparar con las preocupaciones de los ciudadanos catalanes ${ }^{16}$ el temario en la prensa concretó la acción de los partidos en la campaña -tabla IV- evidencia un desencuentro entre las agendas de intereses políticos de la información y los electores:

Tabla IV

Comparación de la agenda informativa y de los asuntos que preocupan a los ciudadanos

\begin{tabular}{|l|c|l|c|}
\hline \multicolumn{1}{|c|}{ Temario de la información } & $\mathbf{( \% )}$ & Preocupaciones de los ciudadanos & (\%) \\
\hline Cultura & 0 & Educación/ cultura/ investigación & 7,1 \\
\hline Educación & 1 & & 18,8 \\
\hline Economía & 4 & Desempleo/ Precariedad Laboral & 4,8 \\
\cline { 2 - 4 } & & Bajo nivel salarial/ coste de la vida & 4,8 \\
\hline
\end{tabular}

${ }^{16}$ Respuesta a la pregunta número 1 “Quin/screu que són els principals problemas que té actualment Catalunya?” del Baròmetro de Opinión Política de la Generalitat de Cataluña de octubre de 2006. (Generalitat de Cataluña, 2006a:6). 


\begin{tabular}{|c|c|c|c|}
\hline Temario de la información & $(\%)$ & Preocupaciones de los ciudadanos & $(\%)$ \\
\hline \multirow[t]{3}{*}{ Economía } & \multirow[t]{3}{*}{4} & Funcionamiento de la economía & 4,4 \\
\hline & & Más dinero/ Nueva financiación & 3,3 \\
\hline & & Excesiva presión fiscal & 2,8 \\
\hline \multirow[t]{2}{*}{ Infraestructuras } & \multirow[t]{2}{*}{2} & Falta de infraestructuras y transporte & 10,3 \\
\hline & & Servicios deficientes & 2,1 \\
\hline Inmigración & 3 & Inmigración & 38,0 \\
\hline Medioambiente & 0 & & \\
\hline \multirow[t]{3}{*}{ Nacionalismo } & \multirow[t]{3}{*}{12} & Crisis de la identidad catalana & 6,4 \\
\hline & & Relaciones Cataluña-España & 4,3 \\
\hline & & Nuevo Estatuto/ Más autogobierno & 3,9 \\
\hline NNTT & 0 & & \\
\hline Salud & 0 & Sanidad y Seguridad Social & 6,9 \\
\hline \multirow[t]{2}{*}{ Seguridad } & \multirow[t]{2}{*}{2} & Inseguridad ciudadana & 12,1 \\
\hline & & Incivismo y violencia & 2,9 \\
\hline Social & 3 & & \\
\hline Vivienda & 0 & Acceso a la vivienda & 22,1 \\
\hline Acuerdos postelectorales & 15 & \multirow[t]{2}{*}{ Mejora política social } & \multirow[t]{2}{*}{7,2} \\
\hline Campaña & 39 & & \\
\hline Candidato & 11 & \multirow[t]{3}{*}{ Insatisfacción con la política } & \multirow[t]{3}{*}{10,7} \\
\hline Partido & 5 & & \\
\hline Tripartito & 2 & & \\
\hline
\end{tabular}

Los asuntos que, según la opinión manifestada en el Barómetro de la Generalitat de Cataluña, los ciudadanos consideran los principales problemas que tiene esta comunidad autónoma, aparecen muy excepcionalmente en la agenda informativa de la campaña: la inmigración que preocupa a 40 de cada 100 catalanes aparece sólo en 3 de cada 100 informaciones, mientras que el acceso a la vivienda o las dificultades laborales que preocupan a 20 de cada 100 se tratan muy raramente. En paralelo, los temas más frecuentes en las informaciones sobre actos de los partidos -Acuerdos postelectorales, Campaña o Candidato- tienen para los electores un interés muy inferior que se manifiesta además con un enfoque negativo: la expectativa de mejora política social y la insatisfacción con la política. 
Por categorías los asuntos de Representación política interesan menos a los ciudadanos que a los periódicos, mientras que los de Acción política los tratan los periódicos muy por debajo de los intereses sociales. sutiles:

Comparar el temario de los periódicos catalanes y los nacionales -Tabla V- muestra diferencias muy

Tabla V

Comparación del temario entre los diarios catalanes y los nacionales

\begin{tabular}{|c|c|c|c|c|}
\hline & \multicolumn{2}{|c|}{ Prensa Nacional } & \multicolumn{2}{|c|}{ Prensa Catalana } \\
\hline & $\mathbf{T}$ & (\%) & $\mathbf{T}$ & $(\%)$ \\
\hline Cultura & 2 & 0 & 3 & 1 \\
\hline Economía & 17 & 4 & 18 & 3 \\
\hline Educación & 4 & 1 & 2 & 0 \\
\hline Infraestructuras & 12 & 3 & 7 & 2 \\
\hline Inmigración & 13 & 3 & 16 & 3 \\
\hline Medioambiente & 2 & 0 & 3 & 1 \\
\hline Nacionalismo & 72 & 16 & 50 & 9 \\
\hline NNTT & 1 & 0 & 1 & 0 \\
\hline Salud & 0 & 0 & 4 & 1 \\
\hline Seguridad & 7 & 2 & 8 & 1 \\
\hline Social & 14 & 3 & 19 & 4 \\
\hline Vivienda & 1 & 0 & 1 & 0 \\
\hline Tripartito & 12 & 3 & 9 & 2 \\
\hline \multicolumn{4}{|c|}{ (35) } & (27) \\
\hline Acuerdos postelectorales & 76 & 17 & 74 & 14 \\
\hline Campaña & 160 & 35 & 228 & 42 \\
\hline Candidato & 30 & 7 & 76 & 14 \\
\hline Partido & 28 & 6 & 18 & 3 \\
\hline \multicolumn{4}{|c|}{$(65)$} & (73) \\
\hline Total & 451 & - & 537 & - \\
\hline
\end{tabular}

44 doxa comunicación n. ${ }^{\circ} 5$ 
En los dos casos el tema que más trataron los periódicos fue la propia campaña, aunque en mayor proporción los catalanes. El segundo tema más presentado en los diarios nacionales fueron los posibles pactos postelectorales, que también fue el segundo más frecuente en los catalanes aunque en proporción menor y empatando con la atención a los candidatos. El tercer tema más tratado también coincide: el Nacionalismo; pero en los nacionales tuvo un peso mucho mayor. El resto de los temas se trataron escasamente -nunca superaron el $10 \%$ de los textos- y con diferencias poco relevantes entre las cabeceras de ámbito catalán y las de ámbito nacional.

Al agrupar en categorías los temas se repite el patrón general de presentar en más textos asuntos de representación política que de acción política. Aunque en los catalanes la representación tiene más peso: el $73 \%$ de la cobertura de los catalanes se ocupó de aspectos relacionados con la representación política y en los nacionales el 65\%. Proporcionalmente los nacionales atendieron cuestiones de acción política en el $32 \%$ de sus textos y los catalanes un $25 \%$.

\subsection{Atención a los partidos}

La campaña que presentaron los diarios analizados -según muestra la tabla VI- se centró en los cinco partidos que en la legislatura 2003-2006 habían obtenido escaños en el Parlamento catalán: la presencia de CiU, PSC, ERC, PP e ICV-EUiA supone el $85 \%$ de la cobertura total. Un dato cuantitativo que se refuerza cualitativamente al considerar que el grueso de la cobertura clasificada como protagonista genérico fueran piezas del género de la encuesta periodística, en las que al plantear a los partidos preguntas cerradas sobre un asunto sólo se incluía a los cinco mencionados.

La presencia de partidos extraparlamentarios fue testimonial, y la copó C-CdC.

Tabla VI

Protagonismo en la información sobre la actividad de los partidos

\begin{tabular}{|l|c|c|}
\cline { 2 - 3 } \multicolumn{1}{c|}{} & Módulos & (\%) \\
\hline CiU & 4.177 & 22 \\
\hline PSC & 4.221 & 23 \\
\hline ERC & 3.216 & 17 \\
\hline PP & 2.723 & 14 \\
\hline ICV-EUiA & 1.754 & 9 \\
\hline C-CdC & 332 & 2 \\
\hline Otros & 76 & 0 \\
\hline Genérico & 2.533 & 13 \\
\hline Total & 19.032 & - \\
\hline
\end{tabular}


Dentro de esta mayor atención a los partidos parlamentarios, destaca la prestada a CiU y PSC que juntos, y en proporciones muy similares, suman casi la mitad de la cobertura total.

Comparando las proporciones de protagonismo en la información de la campaña y de voto en las elecciones de los partidos que obtuvieron representación parlamentaria en las elecciones del 1 de noviembre $^{17}$-Tabla VII- aparecen nuevas diferencias entre los intereses de los ciudadanos y la prensa:

Tabla VII

Comparación entre los procentajes de voto y de cobertura total

\begin{tabular}{|l|c|c|}
\cline { 2 - 3 } \multicolumn{1}{c|}{} & \% voto & \% cobertura \\
\hline CiU & 31,52 & 22 \\
\hline PSC & 26,82 & 23 \\
\hline ERC & 14,03 & 17 \\
\hline PP & 10,65 & 14 \\
\hline ICV-EUiA & 9,52 & 9 \\
\hline C-CdC & 3,03 & 2 \\
\hline
\end{tabular}

Sólo en el caso de ICV-EUiA y C- CdC los porcentajes de voto y de cobertura son próximos. En el caso de CiU y de PSC el voto fue superior a la cobertura total obtenida; muy superior en el caso de CiU partido al que la prensa atendió diez puntos menos que el voto ciudadano. Los dos extremos del arco parlamentario catalán -ERC y PP- recibieron, en cambio, una atención superior a sus resultados electorales: cercana a los tres puntos en los dos casos.

La agenda también presenta algunas diferencias considerada desde el punto de vista del ámbito geográfico:

Tabla VIII

Protagonismo según el lugar de publicación

\begin{tabular}{|l|c|c|c|c|}
\cline { 2 - 5 } \multicolumn{1}{c|}{} & \multicolumn{2}{c|}{ Prensa Nacional } & \multicolumn{2}{c|}{ Prensa Catalana } \\
\cline { 2 - 5 } \multicolumn{1}{c|}{} & $\mathbf{M}$ & $\mathbf{( \% )}$ & $\mathbf{M}$ & $\mathbf{( \% )}$ \\
\hline CiU & 1.323 & 24 & 2.854 & 21 \\
\hline PSC & 1.248 & 23 & 2.973 & 22 \\
\hline
\end{tabular}

17 (Generalitat de Cataluña, 2006c). 


\begin{tabular}{|l|c|r|r|r|}
\cline { 2 - 5 } \multicolumn{1}{c|}{} & \multicolumn{2}{c|}{ Prensa Nacional } & \multicolumn{2}{c|}{ Prensa Catalana } \\
\cline { 2 - 5 } \multicolumn{1}{c|}{} & M & $\mathbf{( \% )}$ & M & (\%) \\
\hline ERC & 907 & 17 & 2.309 & 17 \\
\hline PP & 732 & 13 & 1.991 & 15 \\
\hline ICV-EUiA & 501 & 9 & 1.253 & 9 \\
\hline C-CdC & 290 & 5 & 42 & 0 \\
\hline Otros & 44 & 1 & 32 & 0 \\
\hline Genérico & 421 & 8 & 2.112 & 16 \\
\hline Total & 5.466 & - & 13.566 & - \\
\hline
\end{tabular}

El caso más significativo es el de C- CdC cuya reducida presencia mediática depende casi exclusivamente de los diarios nacionales. También CiU aparece más en los periódicos nacionales, mientras que los catalanes atendieron más al PP y publicaron más información ocupándose de varios partidos a la vez.

No hay diferencias relevantes en el caso de PSC, ERC e ICV-EUiA.

Al comparar los porcentajes de voto que obtuvieron los partidos con su peso en la agenda de los diarios nacionales y catalanes, la única diferencia significativa respecto a los datos generales expuestos en el marco de la tabla VII, es el caso de C-CdC: la tabla IX muestra como los diarios nacionales, además de ser los únicos que se ocupan de este partido, le presentan en proporción superior a la de los votos que consiguió.

En ninguno de los otros partidos la diferencia entre el tratamiento nacional y el catalán supera los tres puntos.

Tabla IX

Comparación del procentaje de voto y los de cobertura según el ámbito de difusión

\begin{tabular}{|l|c|c|c|}
\cline { 3 - 4 } \multicolumn{1}{c|}{} & \multirow{2}{*}{ \% voto } & \multicolumn{2}{c|}{ \% cobertura } \\
\cline { 3 - 4 } \multicolumn{1}{c|}{} & & P. Nacional & P. Catalana \\
\hline CiU & 31,5 & 24 & 21 \\
\hline PSC & 26,8 & 23 & 22 \\
\hline ERC & 14,03 & 17 & 17 \\
\hline PP & 10,6 & 13 & 15 \\
\hline ICV-EUiA & 9,5 & 9 & 9 \\
\hline C-CdC & 3,03 & 5 & 0 \\
\hline
\end{tabular}




\section{Perfil de la campaña: personalización, espectacularización y tipo de mensajes}

\subsection{Personalización}

La tabla X muestra como la prensa personalizó notablemente la campaña de las elecciones autonómicas catalanas de noviembre de 2006: dos tercios de toda la cobertura se centró en los candidatos, el doble de la atención que los diarios prestaron a los partidos.

Tabla X

Grado de personalización de la cobertura total

\begin{tabular}{|l|c|c|}
\cline { 2 - 3 } \multicolumn{1}{c|}{} & Módulos & (\%) \\
\hline Candidato & 12.356 & 65 \\
\hline Partido & 6.676 & 35 \\
\hline Total & 19.032 & - \\
\hline
\end{tabular}

La tabla XI muestra como en los dos ámbitos geográficos se repite la personalización, aunque resulta mucho mayor en la prensa catalana:

Tabla XI

Grado de personalización según ámbito geográfico

\begin{tabular}{|l|c|c|c|c|}
\cline { 2 - 5 } \multicolumn{1}{c|}{} & \multicolumn{2}{c|}{ Prensa Nacional } & \multicolumn{2}{c|}{ Prensa Catalana } \\
\cline { 2 - 5 } \multicolumn{1}{c|}{} & Módulos & $\mathbf{( \% )}$ & Módulos & $\mathbf{( \% )}$ \\
\hline Candidato & 3.081 & 56 & 9.275 & 68 \\
\hline Partido & 2.385 & 44 & 4.291 & 32 \\
\hline Total & 5.466 & - & 13.566 & - \\
\hline
\end{tabular}

En los diarios nacionales la proporción de cobertura de candidatos fue mayor que la de sus partidos, si bien se trata de una diferencia de sólo seis puntos, rondando el 50\%. En cambio en los diarios catalanes la diferencia es mayor: de los candidatos se ocupó el doble de información que de los partidos.

ICV-EUiA es el partido cuya campaña los diarios presentaron más personalizada: ocho de cada diez textos sobre este partido se centran en Joan Saura. CiU y ERC son los segundo y tercer partido más personalizado: de Artur Mas y Josep Ll. Carod -Robira ocupan en torno a tres cuartos de toda la información sobre sus formaciones. PSC y PP presentan una personalización más moderada- en torno al $60 \%$ de los textos que informan sobre ellos se centran en José Montilla y Josep Piqué. 
Sólo en el caso de los partidos a los que los periódicos prestaron una menor atención- C-CdC y "Otros" -y en los textos aparece más de un partido, se presenta al partido en bloque más veces que al cabeza de lista:

Tabla XII

Grado de personalización según partidos

\begin{tabular}{|c|c|c|c|c|c|}
\hline & \multicolumn{2}{|c|}{ Candidato } & \multicolumn{2}{|c|}{ Partido } & \multirow{2}{*}{ Total } \\
\hline & M & $(\%)$ & $\mathbf{M}$ & (\%) & \\
\hline CiU & 3.167 & 76 & 1.010 & 24 & 4.177 \\
\hline PSC & 2.592 & 61 & 1.629 & 39 & 4.221 \\
\hline ERC & 2.269 & 71 & 947 & 29 & 3.216 \\
\hline PP & 1.604 & 59 & 1.119 & 41 & 2.723 \\
\hline ICV-EUiA & 1.462 & 83 & 292 & 17 & 1.754 \\
\hline C-CdC & 99 & 30 & 233 & 70 & 332 \\
\hline Otros & - & - & 76 & 100 & 76 \\
\hline Genérico & 1.163 & 46 & 1.370 & 54 & 2.533 \\
\hline
\end{tabular}

La tabla XIII profundiza en este dato de la personalización generalizada analizando en qué medida los líderes nacionales y los partidos nacionales estuvieron presentes en la campaña autonómica catalana de los tres partidos que tienen una dimensión nacional:

Tabla XIII

Detalle de la personalización en los partidos con dimensión nacional

\begin{tabular}{|l|c|c|c|c|c|c|}
\cline { 2 - 7 } \multicolumn{1}{c|}{} & \multicolumn{2}{c|}{ PSC } & \multicolumn{2}{c|}{ PP } & \multicolumn{2}{c|}{ ICV-EUiA } \\
\cline { 2 - 7 } \multicolumn{1}{c|}{} & M & $\mathbf{( \% )}$ & M & $\mathbf{( \% )}$ & M & (\%) \\
\hline Candidato & 2.592 & 61 & 1.604 & 59 & 1.462 & 84 \\
\hline Partido catalán & 709 & 17 & 362 & 13 & 278 & 16 \\
\hline Lider nacional & 914 & 22 & 607 & 22 & 10 & 0 \\
\hline Partido nacional & 6 & 0 & 150 & 6 & 4 & 0 \\
\hline Total & 4221 & - & 2.723 & - & 1.754 & - \\
\hline
\end{tabular}

La personalización resulta ser doble en la cobertura de PSC y PP: no sólo sus cabezas de lista reciben más atención que los partidos globalmente considerados sino que además, al detallar el contenido de 
su variable "Partido" resulta que los líderes nacionales son los más tratados. José Luís Rodríguez Zapatero y Mariano Rajoy tienen en la representación periodística de la campaña catalana más visibilidad que el conjunto de las dimensiones nacional y catalana de sus partidos.

Ni el líder ni la dimensión nacionales de ICV-EUiA tienen una presencia significativa en la información sobre la campaña de este partido. Se debe a la focalización de los periódicos en torno a Joan Saura, y la estructura de IU.

\subsection{Espectacularización}

En la presentación que hicieron los periódicos de la campaña de los partidos lo espectacular tiene un peso importante como muestra la tabla siguiente.

Tabla XIV

Grado de espectacularización de la cobertura total

\begin{tabular}{|l|c|c|}
\cline { 2 - 3 } \multicolumn{1}{c|}{} & Módulos & (\%) \\
\hline P. real & 10.759 & 57 \\
\hline P. espectáculo & 8.273 & 43 \\
\hline Total & 19.032 & - \\
\hline
\end{tabular}

Es cierto que los asuntos calificados como "Política real" son la mayoría, pero que las manifestaciones espectaculares de la política ocupen casi la mitad de la cobertura, supone que lo excepcional y lo anecdótico recibieron una atención desmesurada.

Tanto en los periódicos nacionales como en los catalanes la política espectáculo supera el $40 \%$ de la cobertura total. Aunque los catalanes se ocupan ligeramente más de asuntos de política real:

Tabla XV

Grado de espectacularización según ámbito geográfico

\begin{tabular}{|l|c|c|c|c|}
\cline { 2 - 5 } \multicolumn{1}{c|}{} & \multicolumn{2}{c|}{ Prensa Nacional } & \multicolumn{2}{c|}{ Prensa Catalana } \\
\cline { 2 - 5 } \multicolumn{1}{c|}{} & Módulos & $\mathbf{( \% )}$ & Módulos & $\mathbf{( \% )}$ \\
\hline P. real & 2.956 & 54 & 7.803 & 58 \\
\hline P. espectáculo & 2.510 & 46 & 5.763 & 42 \\
\hline Total & 5.466 & - & 13.566 & - \\
\hline
\end{tabular}


La tabla, que a continuación se expone, muestra las diferentes proporciones de Política real y de Política espectáculo en la representación de la campaña de cada partido:

Tabla XVI

Grado de espectacularización según partidos

\begin{tabular}{|l|c|c|c|c|c|}
\cline { 2 - 6 } \multicolumn{1}{c|}{} & \multicolumn{2}{c|}{ P. real } & \multicolumn{2}{c|}{ P. espectáculo } & Total \\
\cline { 2 - 6 } \multicolumn{1}{c|}{} & Módulos & $\mathbf{( \% )}$ & Módulos & $\mathbf{( \% )}$ & Módulos \\
\hline CiU & 2.157 & 52 & 2.020 & 48 & 4.177 \\
\hline PSC & 2.251 & 53 & 1.970 & 47 & 4.221 \\
\hline ERC & 2.053 & 64 & 1.163 & 36 & 3.216 \\
\hline PP & 1.620 & 60 & 1.103 & 40 & 2.723 \\
\hline ICV-EUiA & 1.019 & 58 & 735 & 42 & 1.754 \\
\hline C-CdC & 219 & 66 & 113 & 34 & 332 \\
\hline Otros & 46 & 61 & 30 & 39 & 76 \\
\hline Genérico & 1.394 & 55 & 1.139 & 45 & 2.533 \\
\hline
\end{tabular}

Las campañas de CiU y PSC son las que los periódicos presentaron en mayor medida desde el punto de vista del espectáculo: casi la mitad del tratamiento de estos dos partidos se centra en aspectos anecdóticos o sensacionales. ICV-EUiA y PP ocupan un lugar intermedio con porcentajes de espectacularización en torno al 40\%. C-CdC y ERC son los que más veces aparecen en el marco de asuntos de política real.

\subsection{Contenido de los mensajes}

La mayor parte de los titulares de los textos que informan sobre los partidos en campaña son mensajes emitidos por los partidos: afirmaciones, ataques, defensas y propuestas suman el $70 \%$, frente al $30 \%$ de titulares en los que los propios diarios explican un asunto.

Al tomar los partidos la palabra lo más frecuente es que ataquen -un cuarto del total de los mensajes- lo segundo más frecuente es que hagan alguna afirmación, y lo tercero que hagan alguna promesa electoral. Raramente se defienden de algún ataque recibido. 
Tabla XVII

Perfil de los argumentos mostrados en la cobertura total

\begin{tabular}{|c|c|c|}
\hline & & \multirow[b]{2}{*}{$(\%)$} \\
\hline & Textos & \\
\hline Afirmación & 209 & 21 \\
\hline Ataque & 245 & 25 \\
\hline Defensa & 39 & 4 \\
\hline Propuesta & 194 & 20 \\
\hline \multicolumn{3}{|r|}{$(70)$} \\
\hline Descripción & 301 & 30 \\
\hline Total & 988 & - \\
\hline
\end{tabular}

En la prensa nacional los partidos son con más frecuencia sujetos de sus mensajes que en la prensa catalana. Aquí podemos observar concreta las diferencias en el tipo de mensaje:

Tabla XVIII

Perfil de los argumentos mostrados según ámbito geográfico

\begin{tabular}{|l|c|c|c|c|}
\cline { 2 - 5 } \multicolumn{1}{c|}{} & \multicolumn{2}{c|}{ Prensa Nacional } & \multicolumn{2}{c|}{ Prensa Catalana } \\
\cline { 2 - 5 } \multicolumn{1}{c|}{} & Textos & $\mathbf{( \% )}$ & Textos & $\mathbf{( \% )}$ \\
\hline Afirmación & 69 & 15 & 140 & 26 \\
\hline Ataque & 152 & 34 & 93 & 17 \\
\hline Defensa & 20 & 4 & 19 & 4 \\
\hline Propuesta & 109 & 24 & 85 & 16 \\
\hline Descripción & 101 & 22 & 200 & 37 \\
\hline \multicolumn{5}{|l|}{} \\
\hline Total & 451 & - & 537 & - \\
\hline
\end{tabular}

Los diarios de ámbito nacional representan una campaña más agresiva que los catalanes, llevando a los titulares con más frecuencia un ataque -una de cada tres veces. En cambio los catalanes titulan la mayor parte de las veces con una afirmación general. 
Ataques y promesas electorales son, con poca diferencia, el segundo y tercer tipo de mensaje más frecuente en la prensa catalana; mientras que la nacional elige titulares propositivos en un cuarto de sus textos y afirmaciones en algo más de un décimo.

Los titulares defensivos son muy excepcionales.

La tabla XIX muestra como, considerando las diferencias entre partidos, ICV-EUiA, ERC y C-CdC son los que más veces aparecen como sujetos de los titulares que les tratan. PP, CiU y PSC ocupan un lugar intermedio, mientras que al representar partidos menores, o al titular textos sobre varios partidos hay un equilibrio mayor entre toma de la palabra por los partidos y descripción por parte de los periódicos.

Tabla XIX

Perfil de los argumentos mostrados en la cobertura de cada partido

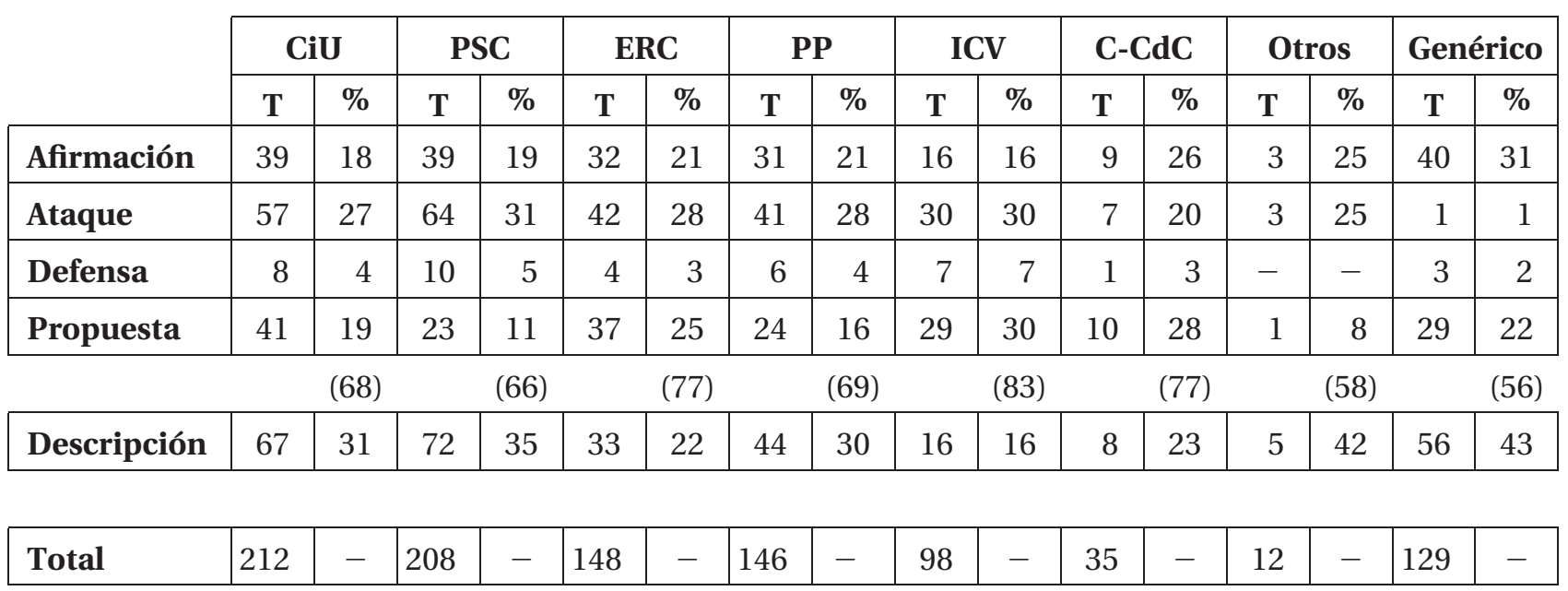

El ataque es el tipo de mensaje concreto con que más veces se titulan los textos sobre CiU, PSC, ERC y PP. El predominio de ataques es más marcado en la representación de PSC y CiU y más sutil en ERC y PP. También los titulares con ataques de ICV-EUiA son numerosos, pero su peso queda matizado al suponer la misma proporción que los titulares con propuestas.

Sólo en el caso de C-CdC el tipo de mensaje más frecuente es la propuesta. 


\section{Conclusiones}

Los resultados hasta aquí expuestos llevan a las siguientes conclusiones:

A) Sobre el contenido de la información electoral

1. Los diarios presentaron más actividades electoralistas de los partidos que informaciones de contexto que orientaran a los votantes: seis de cada diez textos se ocupaban de acciones electoralistas y cuatro del contexto.

2. No se produjeron diferencias relevantes en el foco de interés de los diarios según su ámbito de distribución: aunque los diarios regionales catalanes dedicaron a sus elecciones autonómicas el triple de superficie que los nacionales, las diferencias en la atención que prestaron a los actos de los partidos o al contexto no son significativas cumpliéndose en los dos casos la pauta expuesta en la conclusión 1.

B) Sobre la agenda que la prensa estableció al informar de la campaña de los partidos.

3. Cuatro de los 17 temas encontrados concentran tres cuartas partes de la atención informativa: "Campaña", "Acuerdos postelectorales", "Nacionalismo" y "Candidato". De entre los cuatro el más frecuente es "Campaña", tema del que se ocupa el $40 \%$ de toda la información, los otros tres rondan el 10\%, y de los 13 restantes ninguno supera el $10 \%$.

4. Agrupando los temas por categorías resulta que al presentar la campaña los periódicos atienden más a cuestiones de "Representación política" -en siete de cada diez textos- que a cuestiones de "Acción política".

5. Hay un claro desencuentro entre los temas presentados por los diarios y las preocupaciones manifestadas por los electores: a los temas por los que los catalanes declaran tener mayor preocupación -inmigración, vivienda o las dificultades laborales- los periódicos les dedican una atención marginal al informar de la campaña. En paralelo los temas más tratados por los diarios despiertan en los ciudadanos de Cataluña interés escaso que además tiene un matiz negativo.

Por categorías, los diarios sobredimensionan la "Representación política" respecto a los intereses ciudadanos y minusvaloran la "Acción política"

6. Tanto los diarios de ámbito catalán, como los nacionales, coinciden con la pauta general presentada en la conclusión 4: dedican la mayor parte de sus temarios a "Campaña", "Acuerdos postelectorales", "Nacionalismo" y "Candidato". Las diferencias son moderadas, y se concretan en una mayor atención de los catalanes a la "Campaña" y de los nacionales al "Nacionalismo". 
Por categorías, los diarios de los dos ámbitos geográficos publican más información sobre "Representación política" aunque la proporción es mayor en los catalanes.

7. Los cinco partidos que habían tenido representación parlamentaria en la legislatura 20032006 coparon la atención de la prensa durante la campaña electoral: nueve de cada diez textos. De entre los extraparlamentarios sólo es apreciable el tratamiento del partido de nueva creación C-CdC.

La única diferencia significativa en la presencia de los partidos entre los diarios catalanes y los nacionales es C-CdC, que solo tiene presencia en la información de los diarios nacionales. Ninguna de las variaciones en el resto de los partidos es significativa.

8. Tampoco coincidieron ciudadanos y periódicos en su interés por los partidos que obtuvieron escaños el 1 de noviembre: CiU y PSC tuvieron en la información una presencia inferior a su porcentaje de voto, mientras que los extremos del arco parlamentario catalán -ERC y PP consiguieron de los periódicos más atención que de los ciudadanos. Sólo en los casos de ICV- EUiA y C-CdC hubo afinidad entre votos y atención periodística.

C) Sobre el tipo de la campaña que presentaron los diarios:

9. La prensa personalizó en un alto grado la campaña dedicando a los candidatos el doble de información que a los partidos. Y dentro de esta pauta, la proporción de personalización en los diarios catalanes fue ligeramente mayor.

Por partidos, sólo al informar de C-CdC se trata más veces al partido que al candidato, presentando índices de personalización superiores al 50\% PP y PSC, superiores al 70\% CiU y ERC y superiores al $80 \%$ ICV-EUiA.

En el caso de PSC y PP la personalización resulta mayor al descubrir que dentro de "Partido" los líderes nacionales reciben un tratamiento mayor que el resto de su partido en Cataluña y en toda la nación.

10. Los aspectos extravagantes de la campaña catalana recibieron una atención desmesurada por parte de los diarios analizados: cuatro de cada diez textos trataban asuntos de "Política espectáculo" frente a los seis que se ocupaban de asuntos políticos tradicionales. Esta pauta se repite por geografía sin diferencias significativas.

CiU y PSC son los más espectacularizados, C-CdC y ERC los menos.

11. Los mensajes que más frecuentemente se llevaron a los titulares fueron ataques, en una proporción cinco veces mayor que las propuestas propias de una campaña electoral, que resultan el penúltimo tipo de mensaje menos usado para encabezar los textos. La prensa 
nacional tituló la campaña con más mensajes agresivos -el doble que la catalana, pero también con casi el doble de propuestas.

Por partidos PSC, ICV-EUiA y CiU son los que más veces atacan en sus titulares mientras que C-CdC y ERC son los que más veces proponen.

Todo esto permite emitir un juicio crítico sobre la información que la prensa ofreció en la campaña de las Elecciones autonómicas catalanas, proporcionando más información sobre los actos de los partidos que sobre asuntos de contexto que aportaran a los lectores elementos de juicio, los diarios estaban ofreciendo información poco especializada sometida al calendario y a los intereses de los partidos. Al sobredimensionar temas de representación política, frente a los de gestión real y minusvalorar los asuntos que preocupaban a los ciudadanos, los periódicos estaban alejando a los electores de sus representantes. Mientras que al centrar el foco en los líderes en detrimento de las ideas, preferir los enfoques excéntricos frente a los de política real, y primar en los titulares la disputa frente a la propuesta estaban generando una visión cínica y enrarecida del sistema político.

\section{Referencias bibliográficas}

- Benoit, W.L.; Blaney, J.R.; Pier, P.M. (1998): Campaign 96. A Functional Analysis of Acclaiming, Attacking and Defending, Westport, Praeger.

- Berrocal, S. (2004): "Una aproximación a la nueva retórica del líder político televisivo: acciones, cualidades y discurso”, Doxa Comunicación, vol.II , pp. 53-67.

- Berrocal, S.; Abad, L.; Cebrián, E.; Pedreira, E. (2003): “El 'infoentretenimiento’ televisivo. Las elecciones legislativas de 2000 en El Informal, Caiga Quien caiga y Las noticias del Guiñol” en Berrocal, S. (coord.): Comunicación política en televisión y nuevos medios, Barcelona: Ariel.

- CIDE-INCAS (2006): Anàlisi post-electoral a les eleccions al Parlament de Catalunya de l'1 de novembre, Informe de resultats, Barcelona: CEU.

- CIDE-INCAS (2007): Els abstencionistes en les eleccions al Parlament de Catalunya 2006, Informe de resultats, Barcelona: CEU.

- Estudio General de Medios (2006): Resumen general del EGM de febrero a noviembre de 2006 http:// www.aimc.es/aimc.php?izq = egm.swf\&pag_html = si\&op = cuatro\&dch=02egm/24.html (Consultado el 4 de enero de 2006)

- Generalitat de Cataluña (2006a): Baròmetre d'opinió política Octubre 2006, Barcelona: Registre Públic d'Estudis d'Opinió de la Generalitat de Catalunya núm 363 http://www.idescat.net/cat/idescat/estudis opinio/rpeo/R-363.pdf (Consultado el 5 de diciembre de 2006) 
- Generalitat de Cataluña (2006b): Baròmetre d'opinió política novembre 2006, Barcelona: Registre Públic d'Estudis d'Opinió de la Generalitat de Catalunya núm 367 http://www.idescat.net/cat/idescat/estudis opinio/rpeo/R-367.pdf (Consultado el 5 de diciembre de 2006)

- Generalitat de Cataluña (2006c): Eleccions al Parlament de Catalunya 2006. Resultats per territori, Barcelona: http://www10.gencat.net/pls/gov_eleccions/p12.consultar_res_cat?v_tipus_eleccio=A\&v_any_ eleccio $=2006 \& v \_n u m \_e l e c c i o=1$ (Consultado el 22 de diciembre de 2006)

- Gerstlé, J. (2005): La comunicación política Santiago: LOM Ediciones.

- Muñoz-Alonso, A. (1997): Elogio del periódico Madrid: Universidad San Pablo CEU.

- Swanson, D.L. (1999): "El campo de la comunicación política. La democracia centrada en los medios" en Muñoz-Alonso, A.; Rospir, J.L (eds.): Comunicación política, Madrid Universitas, pp. 3-24.

- Wolton, D. (1999): Sobre la comunicación, Madrid: Acento Editorial. 
